\title{
Thoracic Trauma Severity score on admission allows to determine the risk of delayed ARDS in trauma patients with pulmonary contusion
}

\author{
Aurélien Daurat ${ }^{\mathrm{a}}$, Ingrid Millet ${ }^{\mathrm{b}}$, Jean-Paul Roustan ${ }^{\mathrm{a}}$, Camille Maury ${ }^{\mathrm{a}}$, Patrice Taourel ${ }^{\mathrm{b}}$, \\ Samir Jaber ${ }^{c, d}$, Xavier Capdevila ${ }^{\mathrm{a}, \mathrm{d}}$, Jonathan Charbit ${ }^{\mathrm{a}, *}$ \\ a Trauma Intensive and Critical Care Unit, Lapeyronie University Hospital, Montpellier, France \\ ${ }^{\mathrm{b}}$ Department of Radiology, Lapeyronie University Hospital, Montpellier, France \\ ${ }^{\mathrm{c}}$ Intensive Care Unit and Transplantation, Critical Care and Anesthesia Department (DAR B), Saint-Éloi University Hospital, Montpellier, France \\ 'Institut National de la Santé et de la Recherche Médicale, Inserm U1046, Montpellier, France
}

Keywords:

Thoracic Trauma Severity score

Pulmonary contusion

Acute respiratory distress syndrome

Berlin definition

\begin{abstract}
A B S T R A C T
Background: Pulmonary contusion is a major risk factor of acute respiratory distress syndrome (ARDS) in trauma patients. As this complication may appear after a free interval of $24-48 \mathrm{~h}$, detection of patients at risk is essential. The main objective of this study was to assess the performance of the Thoracic Trauma Severity (TTS) score upon admission in predicting delayed ARDS in blunt trauma patients with pulmonary contusion.

Methods: All blunt thoracic trauma patients admitted consecutively to our trauma centre between January 2005 and December 2009 were retrospectively included if they presented a pulmonary contusion on the admission chest computed tomography scan. Main outcome measure was the presence of moderate or severe ARDS $\left(\mathrm{PaO}_{2} / \mathrm{FiO}_{2}\right.$ ratio $\left.\leq 200\right)$ for $48 \mathrm{~h}$ or more. The global ability of the TTS score to predict ARDS was studied by ROC curves with a threshold analysis using a grey zone approach.

Results: Of 329 patients studied (75\% men, mean age 36.9 years [SD 17.8 years], mean Injury Severity Score 21.7 [SD 16.0]), 82 (25\%) presented with ARDS (mean lowest $\mathrm{PaO}_{2} / \mathrm{FiO}_{2}$ ratio of 131 [SD 34]). The area under the ROC curves for the TTS score in predicting ARDS was $0.82(95 \% \mathrm{CI} 0.78-0.86)$ in the overall population. TTS scores between 8 and 12 belonged to the inconclusive grey zone. A TTS score of 13-25 was found to be independent risk factors of ARDS (OR 25.8 [95\% CI 6.7-99.6] $P<0.001$ ).

Conclusions: An extreme TTS score on admission accurately predicts the occurrence of delayed ARDS in blunt thoracic trauma patients affected by pulmonary contusion. This simple score could guide early decision making and management for a non-negligible proportion of this specific population.
\end{abstract}

\section{Background}

Thoracic trauma occurs in more than $50 \%$ of blunt trauma patients [1]. In this setting, pulmonary contusions are one of the most common injuries, found in $30-75 \%$ of these trauma patients [2]. The occurrence of pulmonary contusions was associated with higher mortality in several studies, especially because it frequently evolved to gas exchange impairment, delayed acute respiratory

\footnotetext{
* Corresponding author at: Département d'Anesthésie Réanimation Lapeyronie, Hôpital Lapeyronie, 371 Avenue du Doyen G. Giraud, 34295 Montpellier, France.

E-mail addresses: daurat.aurelien@gmail.com (A. Daurat), i-millet@chu-montpellier.fr (I. Millet),jp-roustan@chu-montpellier.fr (J.-P. Roustan), c-maury@chu-montpellier.fr (C. Maury), p-taourel@chu-montpellier.fr (P. Taourel), s-jaber@chu-montpellier.fr (X. Capdevila), j-charbit@chu-montpellier.fr (J. Charbit).
}

distress syndrome (ARDS) and/or multi-organ failure [3,4]. The pathophysiological pathway is the activation of local and systemic inflammatory mechanisms in which innate immunity plays a key role [5]. It has been well demonstrated that these deleterious mechanisms may appear after a free interval of 24-48 h [6]. Consequently, in trauma patients with pulmonary contusion, initial assessment may underestimate the gravity of the situation whereas respiratory status may worsen during the hours or days following admission [7].

Many scoring systems have been developed in recent years to define injury severity in cases of thoracic trauma and pulmonary contusion [8]. Some of these scores are global, such as the Abbreviated Injury Scale (AIS) and the Injury Severity Score (ISS), but they do not provide detailed analysis of injuries $[9,10]$. On the contrary, some other scores focus only on one injury (e.g. the Wagner score for pulmonary contusion) without considering 
associated injuries [11]. Furthermore, these scores do not consider gas exchange or the patient's condition, which are major determinants in assessing respiratory risk.

In contrast, the Thoracic Trauma Severity (TTS) score is the only one to account for demographic data such as age or respiratory status (ratio between partial pressure of oxygen in arterial blood and inspired fraction of oxygen $\left[\mathrm{PaO}_{2} / \mathrm{FiO}_{2}\right.$ ratio]) in addition to most thoracic injuries (pleural effusions, pulmonary contusions, rib fractures) [12]. TTS score determined on admission was recently associated with increased occurrence of ARDS and mortality in a population of patients with severe thoracic trauma at very high risk of respiratory failure [13]. The performance of the TTS score for predicting ARDS remains therefore to be determined in a less severe trauma population, especially those who had no respiratory distress on admission; these latter patients, who are most at risk of being underestimated, would benefit from specific prophylactic strategies, however. Knowing the specific risk of delayed progression to ARDS induced by pulmonary contusions, we chose to focus our analysis on these specific injuries. Furthermore, there is a need to determine clinically relevant TTS score thresholds to help with screening and appropriate management of this kind of identified population.

The main objective of the present study was therefore to assess the performance of the TTS score on admission for early prediction of ARDS in blunt thoracic trauma patients with pulmonary contusion. A secondary objective was to statistically determine clinically useful thresholds using a grey zone approach.

\section{Methods}

\section{Study design and patients}

The hospital charts of the trauma intensive care unit (ICU) of Lapeyronie University Hospital (Level I Regional Trauma Centre, Montpellier, France) were studied retrospectively from January 2005 to December 2009. This unit receives directly from scene in its emergency room all trauma patients in the area suspected to be severely injured during pre-hospital assessment according to French guidelines [14].

All patients who had blunt thoracic trauma (AIS $\geq 1$ ) were eligible for a computed tomography (CT) scan, and those who had a pulmonary contusion were included. Exclusion criteria were as follows: (1) absence of a chest CT scan within the first $4 \mathrm{~h}$ after admission; (2) data missing from the medical record; (3) admission more than $24 \mathrm{~h}$ after trauma or from another hospital; (4) death within the first $48 \mathrm{~h}$. Because of its retrospective and observational nature, the local ethics committee waived the need for written consent for this study.

\section{Data collection}

Data on age, sex, mechanism and characteristics of injuries, mortality, ICU and hospital length of stay were extracted from the medical records. The ISS, AIS for each body region and the Simplified Acute Physiology Score II (SAPS II) [15] were calculated for all patients. Data on the need for and the duration of mechanical ventilation were also collected. Values for the partial pressure of oxygen in arterial blood $\left(\mathrm{PaO}_{2}\right)$ and the inspiratory fraction of oxygen $\left(\mathrm{FiO}_{2}\right)$ were obtained on admission and daily at $8 \mathrm{am}$ for the first 10 days of hospitalization. If the patient was not intubated, $\mathrm{FiO}_{2}$ was determined using the rules described by Wagstaff et al. [16] $\mathrm{PaO}_{2} / \mathrm{FiO}_{2}$ ratios were then calculated.

\section{Thoracic injuries and TTS score calculation}

For the present study, a retrospective analysis of all chest CT scans performed on admission was done by an independent radiologist (IM), who was blinded to the patient outcome. The size of the pulmonary contusions was assessed as well as associated thoracic injuries (pneumothoraces, hemothoraces, rib fractures, flail chest and atelectasis). From these findings, the TTS score on admission was determined for each patient on a scale ranging from 0 to 25 points as described by Pape et al. [12] (Table 1).

\section{Primary outcome and definition of ARDS}

The main outcome criterion was the occurrence of ARDS during the first 10 days of hospitalization for $48 \mathrm{~h}$ or more. The international Berlin definition for ARDS was used for this purpose [17]. Patients were thus categorized into two groups according to the occurrence (ARDS group) or the absence of ARDS (no ARDS group). This definition implies the presence of bilateral opacities on chest imaging and hypoxia not fully explained by cardiac failure or fluid overload. ARDS was staged as moderate $\left(\mathrm{PaO}_{2} / \mathrm{FiO}_{2}\right.$ between 101 and 200) and severe $\left(\mathrm{PaO}_{2} / \mathrm{FiO}_{2} \leq 100\right)$. Mild ARDS was not considered.

\section{Statistical analysis}

Distribution of continuous variables was assessed by a Shapiro-Wilk test. Normally distributed data were expressed as means (standard deviation [SD]), and non-normally distributed data as medians (interquartile range [IQR]); comparisons between the ARDS and no ARDS groups were performed using the Student's $t$ test or the Mann-Whitney test as appropriate. Qualitative data were expressed as numbers (percentages) and compared using the chi-squared. A receiver operating characteristic (ROC) curve was then drawn to assess the performance of the TTS score on admission in predicting the occurrence of ARDS during hospitalization. As it was clinically relevant to identify patients on admission with no alteration in gas exchange, who would be at risk of developing ARDS subsequently, another ROC curve was drawn for the subgroup of non-hypoxaemic patients on admission $\left(\mathrm{PaO}_{2} / \mathrm{FiO}_{2}>200\right)$ [18]. Areas under the ROC curves (AUC) were expressed with their 95\% confidence interval (CI). Considering that a single cut-off value may not fit all clinical situations, a threshold analysis was performed using a grey zone approach. Thus, for each ROC curve, two thresholds were determined as previously described by Cannesson et al. [19] to obtain a high sensitivity (Se) threshold (i.e. $\geq 90 \%$ ) and a high specificity (Sp) threshold (i.e. $\geq 90 \%$ ). Positive predictive value (PPV) and negative predictive value (NPV) were provided for each

Table 1

Thoracic Trauma Severity (TTS) score.

\begin{tabular}{|c|c|c|c|c|c|}
\hline $\mathrm{PaO}_{2} / \mathrm{FiO}_{2}$ & Rib fracture & Contusion & Pleural involvement & Age (years) & Points \\
\hline$>400$ & 0 & None & None & $<30$ & 0 \\
\hline $300-400$ & $1-3$ & 1 lobe & Pneumothorax & $30-41$ & 1 \\
\hline $200-300$ & 4-6 unilateral & 1 lobe bilateral or 2 lobes unilateral & Unilateral HT or HPT & $42-54$ & 2 \\
\hline $150-200$ & $>3$ bilateral & $<2$ lobes bilateral & HT or HPT bilateral & $55-70$ & 3 \\
\hline$<150$ & Flail chest & $\geq 2$ lobes bilateral & Tension pneumothorax & $>70$ & 5 \\
\hline
\end{tabular}

All categories have to be added to achieve a score ranging from 0 to 25 . HT, haemothorax; HPT, hemopneumothorax. 
threshold with their 95\% CI. From these findings, the overall population was divided a posteriori into three groups using these thresholds: high risk, intermediate risk and low risk. This categorical variable was assessed using multivariable logistic regression to determine whether it was an independent risk factor for ARDS. In this model, all usual severity and shock variables were included. Finally, linear regression was used to assess the strength of association between the lowest $\mathrm{PaO}_{2} / \mathrm{FiO}_{2}$ ratio observed during hospitalization, ventilation or ARDS duration and the TTS score on admission. XLSTAT Pro 7.5.2 (Addinsoft, Brooklyn, NY) was used for statistical analysis. $P \leq 0.05$ was considered significant.

\section{Results}

\section{Patient characteristics}

During the 5-year study period, 1079 trauma patients were admitted to our trauma ICU, of which 619 had a blunt thoracic trauma and were eligible for CT scan analysis. From this population, 84 patients met exclusion criteria (32 indirect or delayed admissions, 18 deaths within first $24 \mathrm{~h}$ and 34 lacks of clinical data or CT scan) and 206 had no pulmonary contusion. The 329 remaining patients were studied; 246 (75\%) were male, the mean age was 36.9 years (SD 17.8), and the mean ISS was 21.7 (SD 16.0; median ISS 20 [IQR 18-25]). Main mechanisms of injury were motor vehicle crashes, bicycle accidents or falls. The characteristics of these patients are summarized in Table 2. In this population, 44 (13\%) patients were hypoxaemic on admission (hypoxaemic subgroup) and 285 (87\%) were not (non-hypoxaemic subgroup). A total of $142(43 \%)$ patients received mechanical ventilation during hospitalization, including 131 (40\%) during initial management (Fig. 1A).

\section{Occurrence of ARDS}

During the 10 days of follow-up, 82 (25\%) patients presented ARDS, 66 (20\%) moderate cases and 16 (5\%) severe cases. ARDS occurred in 34 patients $(41 \%)$ on day $1,15(18 \%)$ on day $2,11(13 \%)$ on day 3, $6(7 \%)$ on day 4 and $16(19 \%)$ on day 5 or later (Fig. 1B). Median time for onset of ARDS was 2.5 days (range, 0-9 days) and median duration of ARDS was 4.5 days (range 2-10 days). The mean lowest $\mathrm{PaO}_{2} / \mathrm{FiO}_{2}$ ratio in these patients was 131 (SD 34). ARDS occurred less in the subgroup who were non-hypoxaemic on admission than the others: $17 \%(48 / 285)$ versus $77 \%(34 / 44)$, respectively $(P<0.001)$.

As expected, duration of ventilation and ICU length of stay were significantly longer for patients in the ARDS group than in the no ARDS group $(P<0.001)$. The mortality rate was also significantly higher in the ARDS group $(P=0.007)$.

\section{TTS score on admission and the occurrence of ARDS}

The TTS score on admission was significantly higher in the ARDS group than in the no ARDS group: 12 (IQR 10-14) versus 8 (IQR 6$10), P<0.001$. This difference was also observed in the nonhypoxaemic subgroup (Fig. 2). The area under the ROC curves for TTS score on admission in predicting ARDS was 0.82 (95\% CI 0.78$0.86)$ in the overall population and $0.78(95 \% \mathrm{Cl} 0.72-0.82)$ in the non-hypoxaemic subgroup (Fig. 2).

Using the grey zone approach, the high sensitivity threshold in the overall population for the prediction of ARDS was $\geq 8$ (Se 90\%

Table 2

Baseline characteristics and outcome of patients.

\begin{tabular}{|c|c|c|c|c|}
\hline & ARDS group $(n=82)$ & No ARDS group $(n=247)$ & Total $(N=329)$ & $P$ \\
\hline Age (years) & $36.0 \pm 15.9$ & $37.2 \pm 18.3$ & $36.9 \pm 17.8$ & 0.59 \\
\hline Age $\geq 55$ years $(\%)$ & $11(13)$ & $35(14)$ & $46(14)$ & 0.99 \\
\hline Male (\%) & $59(72)$ & $187(76)$ & $246(75)$ & 0.59 \\
\hline \multicolumn{5}{|l|}{ Mechanism of injury (\%) } \\
\hline MVC & $55(67)$ & $123(50)$ & $178(54)$ & 0.085 \\
\hline Bicycle & $11(13)$ & $49(20)$ & $60(18)$ & \\
\hline Pedestrian & $4(5)$ & $9(4)$ & $13(4)$ & \\
\hline Fall & $8(10)$ & $45(18)$ & $53(16)$ & \\
\hline Assault & $4(5)$ & $16(6)$ & $20(6)$ & \\
\hline SAPS II & $36.7 \pm 16.7$ & $25.0 \pm 17.1$ & $28.0 \pm 17.7$ & $<0.001$ \\
\hline ISS & $30.2 \pm 16.7$ & $19.0 \pm 14.8$ & $21.7 \pm 16.0$ & $<0.001$ \\
\hline ISS $\geq 16(\%)$ & $68(83)$ & $122(49)$ & $190(58)$ & $<0.001$ \\
\hline $\mathrm{SBP}<90 \mathrm{mmHg}(\%)$ & $56(68)$ & $63(25)$ & $119(36)$ & \\
\hline Severe anatomical injuries & & & & $<0.001$ \\
\hline Chest AIS $\geq 3$ & $54(66)$ & $126(51)$ & $180(55)$ & 0.027 \\
\hline Head AIS $\geq 3$ & $34(41)$ & $28(11)$ & $62(19)$ & $<0.001$ \\
\hline Abdominal AIS $\geq 3$ & $35(42)$ & $62(25)$ & $97(29)$ & 0.004 \\
\hline Extremity AIS $\geq 3$ & $29(35)$ & $52(21)$ & $81(24)$ & 0.014 \\
\hline \multicolumn{5}{|l|}{ Thoracic injuries } \\
\hline HT/HPT bilateral or TPT & $55(67)$ & $88(36)$ & $143(43)$ & $<0.001$ \\
\hline$>3$ ribs bilateral or fail chest & $47(57)$ & $90(36)$ & $137(42)$ & $<0.001$ \\
\hline Pulmonary contusion $\geq 3$ lobes & $53(65)$ & $98(40)$ & $151(46)$ & $<0.001$ \\
\hline TTS score on admission & $12(10-14)$ & $8(6-10)$ & $10(8-16)$ & $<0.001$ \\
\hline \multicolumn{5}{|l|}{ Respiratory status on admission } \\
\hline Mechanical ventilation (\%) & $74(90)$ & $57(23)$ & $142(43)$ & $<0.001$ \\
\hline $\mathrm{PaO}_{2} / \mathrm{FiO}_{2}$ ratio & $213 \pm 138$ & $401 \pm 110$ & $368 \pm 156$ & $<0.001$ \\
\hline $\mathrm{PaO}_{2} / \mathrm{FiO}_{2}$ ratio $\leq 200(\%)$ & $34(41)$ & $10(4)$ & $44(13)$ & $<0.001$ \\
\hline \multicolumn{5}{|l|}{ Outcome } \\
\hline ICU days & $21.6 \pm 21.6$ & $12.4 \pm 14.5$ & $14.7 \pm 17.2$ & $<0.001$ \\
\hline Hospitalization days & $34.0 \pm 39.0$ & $21.7 \pm 21.3$ & $24.8 \pm 27$ & $<0.001$ \\
\hline Mortality (\%) & $13(16)$ & $14(6)$ & $27(8)$ & 0.007 \\
\hline
\end{tabular}

Data are expressed as mean $\pm S D$, median (interquartile range) or as number of patients (percentage) as appropriate. MVC, motor vehicle crash; ISS, Injury Severity Score; AIS, Abbreviated Injury Scale, SBP, systolic blood pressure; HT, haemothorax; HPT, hemopneumothorax; TPT, tension pneumothorax; TTS score, Thoracic Trauma Severity score. 
A

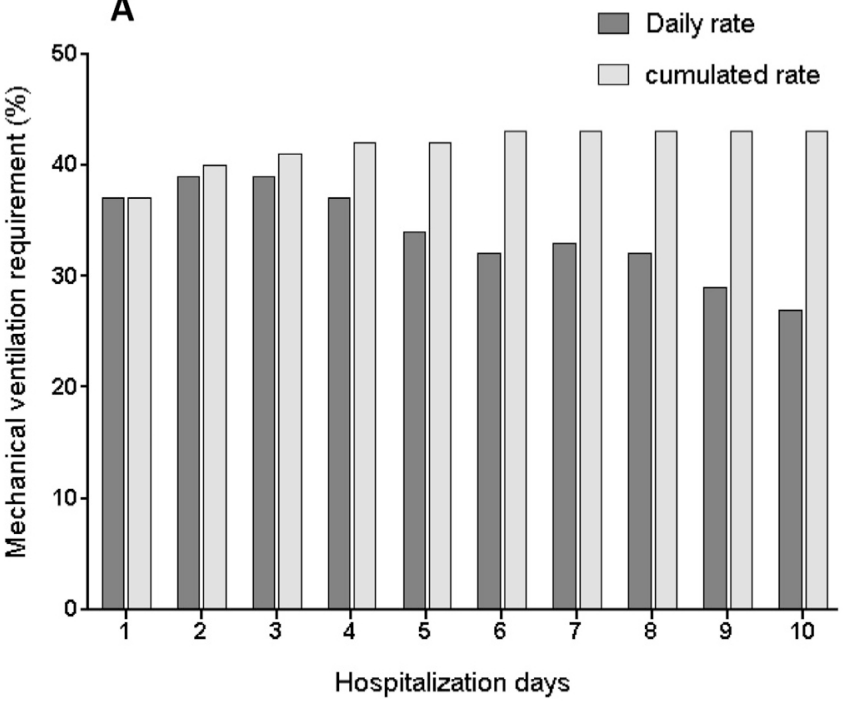

B

Daily rate

cumulated rate

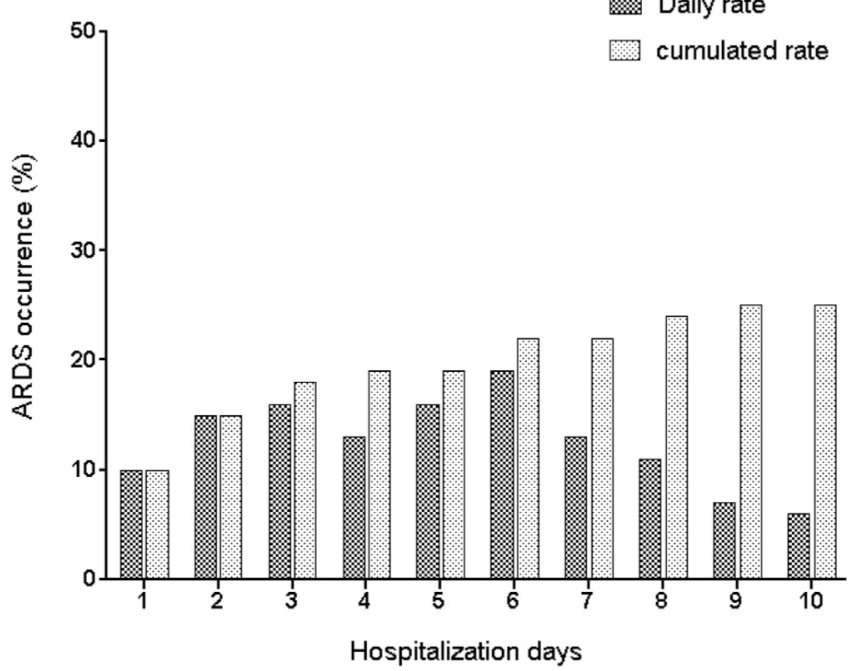

Fig. 1. Mechanical ventilation requirements $(A)$ and occurrence of ARDS (B) $(N=329)$. ARDS, acute respiratory distress syndrome.
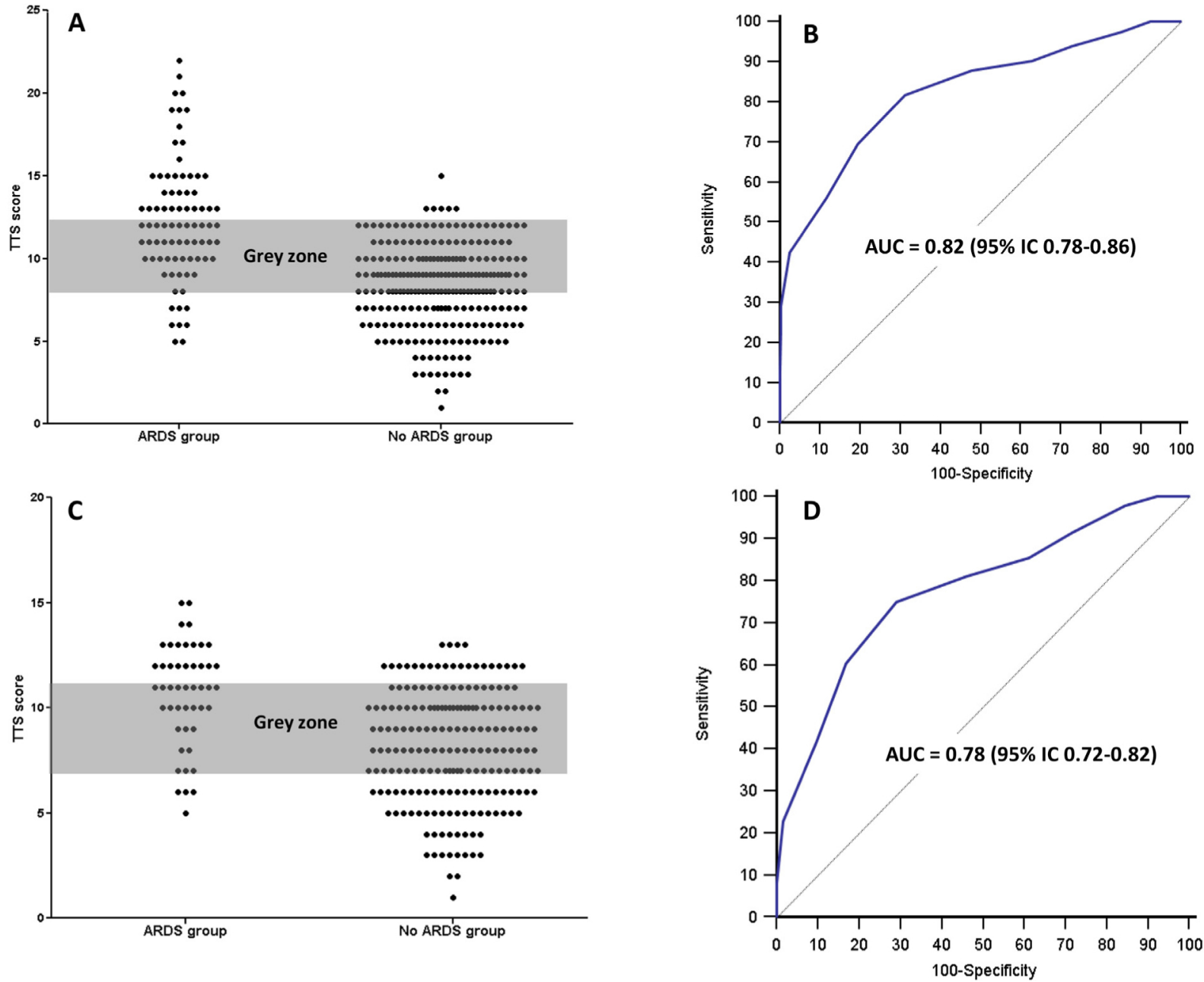

Fig. 2. TTS score on admission and occurrence of ARDS during hospital stay. Dot plot of TTS score values on admission (A) and ROC curve for predicting the occurrence of ARDS (B) in the overall study population $(N=329)$. The inconclusive grey zone is displayed as a grey rectangle for a TTS score between 8 and 12 and included $57 \%$ of the overall population. Dot plot of TTS score values on admission (C) and ROC curve for predicting the occurrence of ARDS (D) in the non-hypoxaemic subgroup ( $n=285$ ). The inconclusive grey zone is displayed as grey rectangle for a TTS score of between 7 and 11 and included $52 \%$ of the subgroup. ARDS, acute respiratory distress syndrome; AUC, area under the curve; ROC curve, receiver operating characteristics. 


\begin{tabular}{|c|c|c|c|c|}
\hline \multirow[t]{2}{*}{ Variables } & \multicolumn{2}{|l|}{ Univariable analysis } & \multicolumn{2}{|c|}{ Multivariable analysis } \\
\hline & OR $(95 \% \mathrm{CI})$ & $P$ value & OR $(95 \% \mathrm{CI})$ & $P$ value \\
\hline \multicolumn{5}{|l|}{ TTS score } \\
\hline $0-7$ & Reference & & Reference & \\
\hline $8-12$ & $3.0(1.3-6.7)$ & 0.040 & $2.1(1.0-3.8)$ & 0.06 \\
\hline $13-25$ & $67.1(21.7-207.2)$ & $<0.001$ & $25.8(6.7-99.6)$ & $<0.001$ \\
\hline $\mathrm{SBP}<90 \mathrm{mmHg}$ & $6.3(3.6-10.9)$ & $<0.001$ & $3.5(1.6-7.9)$ & 0.002 \\
\hline Base deficit $<6 \mathrm{mmol} / \mathrm{L}$ & $3.9(2.3-6.5)$ & $<0.001$ & $1.7(0.8-3.6)$ & 0.18 \\
\hline Head AIS $\geq 3$ & $5.5(3.0-10.0)$ & $<0.001$ & $3.7(1.7-8.1)$ & $<0.001$ \\
\hline Chest AIS $\geq 3$ & $1.8(1.1-3.1)$ & 0.019 & $1.1(0.5-2.2)$ & 0.89 \\
\hline Abdominal AIS $\geq 3$ & $2.2(1.3-3.7)$ & 0.002 & $1.3(0.6-2.8)$ & 0.49 \\
\hline Extremities AIS $\geq 3$ & $2.0(1.2-3.5)$ & 0.009 & $1.2(0.5-2.5)$ & 0.76 \\
\hline Hypoxaemia on admission & $16.8(7.8-36.3)$ & $<0.001$ & $5.5(2.0-14.7)$ & $<0.001$ \\
\hline
\end{tabular}

Multivariate analysis was performed using a logistic regression model (Logit). The usual severity and shock admission variables a priori determined were included in this model to highlight the independent association between risk groups defined by TTS score and ARDS. ARDS, acute respiratory distress syndrome; TTS score, Thoracic Trauma Severity score; OR, odds ratio; CI, confidence interval; AIS, Abbreviated Injury Scale; SBP, systolic blood pressure.

[95\% Cl 82-96\%], Sp 37\% [95\% CI 31-44\%], PPV 32\% [95\% CI 26-39\%], NPV 92\% [95\% CI 85-96\%]) and the high specificity threshold was $\geq 13$ (Se 43\% [95\% CI 32-54\%], Sp 98\% [95\% CI 95-99\%], PPV 85\% [95\% CI 71-94\%], NPV 84\% [95\% CI 79-88\%]). Corresponding thresholds in the non-hypoxaemic subgroup were almost identical; $\geq 7$ (Se 90\% [95\% Cl 80-99\%], Sp 29\% [95\% CI 23-35\%], PPV 15\% [95\% CI 10-20\%], NPV 96\% [95\% CI 91-100\%]) and $\geq 12$ (Se 36\% [95\% CI 18-52\%], Sp 91\% [95\% CI 87-95\%], PPV 36\% [95\% CI 19-52\%], NPV $91 \%$ [95\% CI $87-95 \%]$ ), respectively. The rate of patients who belonged to the grey zone was $57 \%$ in overall population and $51 \%$ the non-hypoxaemic subgroup.

Stratification of population using grey zone thresholds of TTS score (i.e. $0-7,8-12$, and 13-25) was subsequently assessed using a multivariable logistic regression model. The variable "TTS score 13-25" was found to be independently associated with the occurrence of ARDS (Table 3).

\section{TTS score on admission and secondary outcomes}

In further analyses, the TTS score on admission was also significantly associated with severe ARDS, need for mechanical ventilation or length of stay in univariable analysis $(P<0.001)$. Mortality was not found to be statistically associated (Fig. 3).

The linear regression model showed that the TTS score on admission predicted the lowest $\mathrm{PaO}_{2} / \mathrm{FiO}_{2}$ ratio observed subsequently during ICU stays $\left(r^{2}=0.31 ; P<0.001\right)$; each additional point for the TTS score on admission was associated with a decrease in the expected lowest $\mathrm{PaO}_{2} / \mathrm{FiO}_{2}$ ratio of $19.2 \mathrm{mmHg}$. This association was also found for duration of ventilation $\left(r^{2}=0.27 ; P<0.001\right)$ and ARDS duration $\left(r^{2}=0.34 ; P<0.001\right)$; each additional point for the TTS score on admission significantly extended these durations by 0.4 and 1.3 days, respectively.

\section{Discussion}

In the present study of 329 trauma patients with pulmonary contusion admitted to the trauma ICU, 142 (43\%) required mechanical ventilation and $82(25 \%)$ experienced ARDS for $48 \mathrm{~h}$ or more during their hospitalization. The ARDS occurred in $59 \%$ of cases (48/82) after the 24th hour. Our findings demonstrate that the TTS score on admission was significantly associated with the occurrence of ARDS, whether early or delayed; the global ability of the TTS score to predict ARDS was acceptable, with an AUC of 0.82 in the overall population and 0.78 in the non-hypoxaemic subgroup. More interestingly, our analysis also highlighted high sensitivity and specificity thresholds to accurately predict ARDS risk: with a TTS score $<8$, the risk of ARDS may be considered minimal, whereas with a TTS score of 13 or more, the risk of ARDS is nearly certain. This stratification of patients was independently associated with the SDRA risk. Thus, the TTS score allows early and accurate grading of the risk of delayed ARDS for half of this specific population.

Pulmonary contusion is well known to evolve towards respiratory failure, typically after a free interval of $24-48 \mathrm{~h}$ $[6,20]$. This injury primes innate immunity through enhanced reactivity of toll-like receptors 4 (TLR4), which induces exaggerated production of pro-inflammatory mediators well described in the early phase of trauma (e.g. interleukin [IL]-1 $\beta$, IL-6, IL-8) [20]. In the case of secondary insults such as infection or surgical aggression, socalled "second hit", pro-inflammatory mediators induce both local infiltration of neutrophils and systemic inflammation [21]. This proinflammatory cascade leads to ARDS and multi-organ failure, which are strongly associated with increased morbidity and mortality in thoracic trauma [4]. Accordingly, the management of trauma patients with pulmonary contusion remains challenging, especially because the initial clinical evaluation may underestimate the risk of delayed worsening [22]. One of the main strengths of the present series is that we were able to identify and study a homogeneous population, in contrast with many studies on ARDS in the literature. Moreover, statistical analysis was focused on the clinically relevant "non-hypoxaemic" subgroup ( $87 \%$ of our population) because this specific population is at high risk to be misjudged or underestimated. Thus, we observed that $17 \%$ (48/285) of these patients, almost one-fifth, experienced delayed ARDS. This non-negligible rate highlights the need for reliable clinical tools to predict delayed ARDS risk as soon as admission.

Several scoring systems have been developed to predict the outcome of thoracic trauma. Some, such as the Lung Organ Failure Score (LOFS) [23] or the Watkins predictive model [24], determined risk factors associated with ARDS. However, these scores based on retrospective data include variables that are not available on admission such as the amount of fluid or blood products transfused or surgical intervention requirements. Early determination of ARDS risk is accordingly not possible with these scores. Moreover, these scores do not take into account the size of contusions or associated thoracic injuries. Their use during the initial management of patients with thoracic trauma is therefore questionable. Others scores, such as the AIS and the ISS, provide a global and quite empiric estimation of trauma that does not allow precise grading of different thoracic injuries. Many concerns also exist about their usefulness in predicting outcome [25]. Radiological scoring systems (e.g. Miller [26] or Wagner [11] scores) precisely describe the size of contusions, for example, but do not consider injuries association or clinical or respiratory status.

For the reasons discussed earlier, some experts assume that the TTS score is the closest to an ideal scoring system for qualifying 

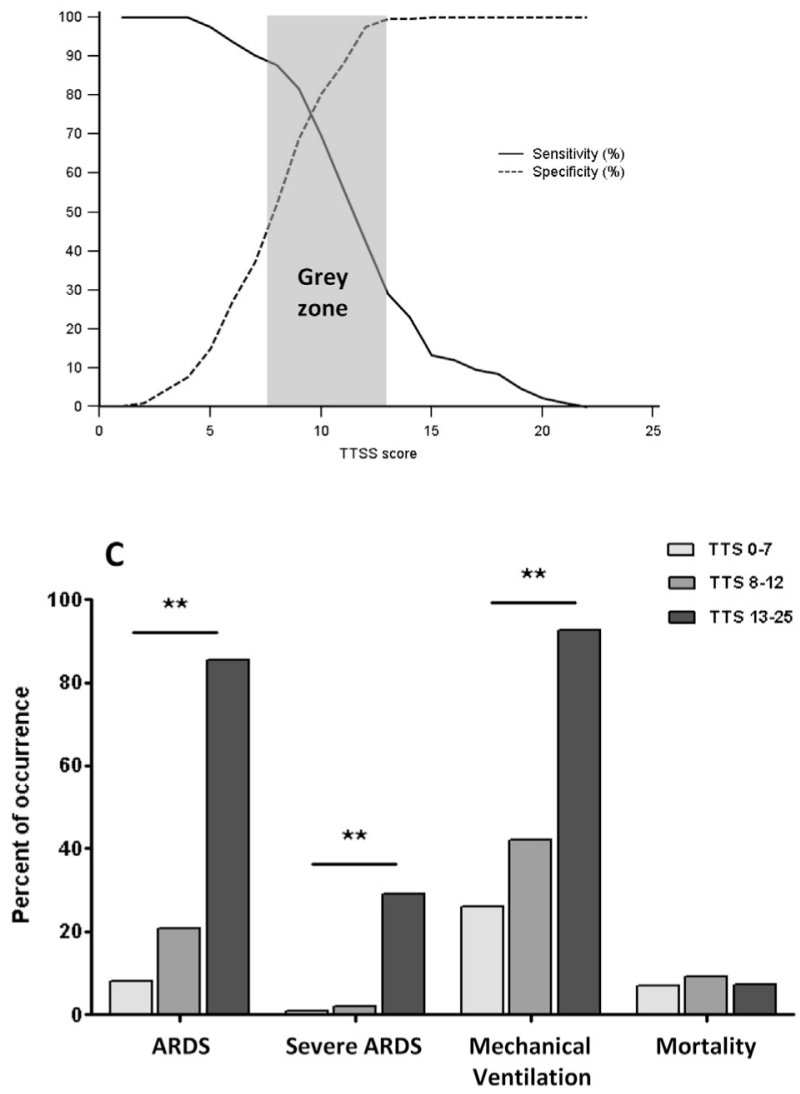
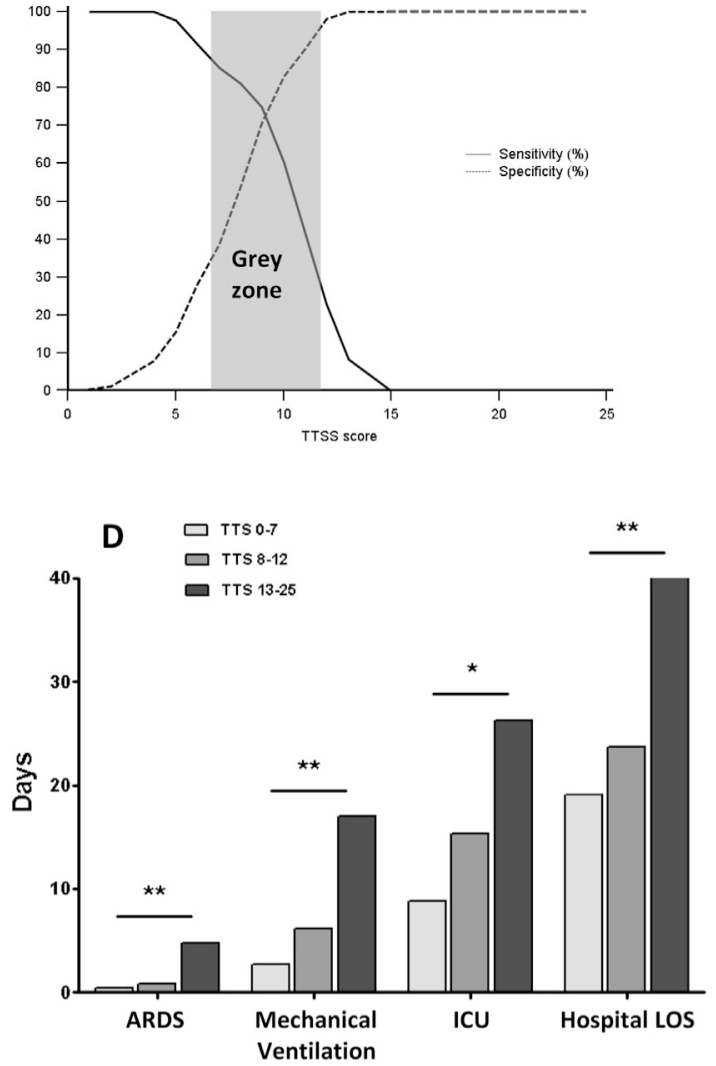

Fig. 3. Grey zone of TTS score and outcome. Two-curve graph showing the sensitivity and specificity of the different values of TTS score to predict ARDS in the overall study population (A) and in the non-hypoxaemic subgroup (B). The inconclusive grey zones are displayed as grey rectangles; the TTS scores were between 8 and 12 and between 7 and 11 and included $57 \%$ and $52 \%$ of patients, respectively. (C) The proportion on mechanical ventilation, the occurrence of severe ARDS, ARDS and mortality according to the TTS score in the overall study population. (D) Duration of mechanical ventilation, ARDS duration, ICU and hospital length of stay according to the TTS score in the overall study population. ARDS, acute respiratory distress syndrome; ICU, intensive care unit; LOS, length of stay. Results of the chi-squared test (C) and one-way ANOVA (D) are displayed as follows: ${ }^{*} P<0.05 ;{ }^{* *} P<0.001$.

thoracic trauma and predicting respiratory impairment [8]. In addition to a semi-quantitative evaluation of most thoracic injuries, the characteristics of the patient (age) as well as gas exchange statuses are considered. This score is moreover easy to calculate on admission in clinical practice by a non-radiologist physician once the initial CT scan has been performed. The TTS score was initially developed by Pape et al. [12] for the prediction of death and global outcome. It was also recently demonstrated that the TTS score was the most robust score for predicting ventilation time, length of stay, and occurrence of ARDS in a population with severe thoracic trauma at high risk of respiratory failure [13]. Nevertheless, its performance has not been studied in a population of less severely injured or in a homogenous population such as patients with pulmonary contusions. Furthermore, the only threshold available in the literature is the best statistical threshold determined using the Youden method [27], which is well known to be unsuitable in clinical practice [28]. Conversely, the grey zone approach defines statistical thresholds that can help the clinician to rule out or predict worsening risk of respiratory problems. Therefore, in the present study we have chosen to define and assess these clinically relevant values for the TTS score on admission in early prediction of ARDS.

Our study thus proves in a large thoracic trauma population affected by pulmonary contusion that the TTS score on admission is significantly associated with the occurrence of ARDS during hospitalization, whether the patients were hypoxaemic or nonhypoxaemic on admission (AUCs $0.82-0.78$; Fig. 2). Using grey zone approach, the high specificity threshold of the TTS score for ARDS prediction was $\geq 13$ (Sp 98\%, PPV 85\%), whereas the high sensitivity threshold was $\geq 8$ (Se $90 \%$, NPV 92\%). It is very interesting to observe that the AUCs and the thresholds were comparable in subgroup of non-hypoxaemic patients on admission. In further analysis, the stratification of patients using previous thresholds was an independent variable in our multivariable predictive model for ARDS (Table 3) and was also associated with secondary outcomes such as the duration of ARDS, mechanical ventilation or hospitalization (Fig. 3). Our findings therefore demonstrated that whatever the initial respiratory status, a clinical approach based on TTS score thresholds allows early identification of a large number of patients at risk of delayed ARDS. This early detection is determinant to guide optimal initial management and preventive measures such as protective mechanical ventilation, restrictive fluid therapy, damage control surgery and strategy of staged interventions $[29,30]$. Non-invasive ventilation and epidural analgesia should also be proposed in nonintubated patients $[31,32]$. In other hand, in patients included in the inconclusive grey zone (i.e. TTS score 8-12), almost the half of studied population, TTS score may fail to assess the risk of delayed respiratory worsening. In these "borderline" patients, the risk of delayed ARDS is however not negligible (Fig. 3). Preventive measures should therefore be considered, particularly in presence of other ARDS risk factors [33].

The present study has some limitations that must be taken into account. The retrospective design may obviously lead to some bias. 
However, selection bias was low because only a few patients were excluded for lack of data. Fluid therapy and sequencing of surgical intervention were not standardised in our centre during the study period, although they are well known to be associated with the occurrence of ARDS $[23,24]$. Ventilatory strategies are not detailed in our series. High tidal volumes and high pressure levels have been indeed strongly associated with respiratory impairment [29]. In addition, the performance of TTS score and its grey zone should be assessed on a larger population and using external validation to be robust and indisputable. Finally, our findings were obtained from patients affected by pulmonary contusion only; these results are therefore probably not generalizable to all patterns of thoracic trauma.

In conclusion, extreme values of TTS score on admission allow us to accurately identify the risk of delayed ARDS at an early stage in almost half of trauma patients affected by pulmonary contusion. A TTS score $\geq \mathbf{1 3}$ was highly predictive of delayed ARDS, whereas a score $\leq 7$ identified low-risk patients. This readily available score on initial assessment helps in screening for delayed risk of respiratory impairment, especially in patients who do not present initial respiratory impairments.

\section{Conflict of interest}

All the authors declare that they do not have any financial and personal relationships with people or organisations that can inappropriately influence the work.

There is no professional or other personal interest of any nature or kind in any product, service and/or company that could be construed as influencing the position.

\section{References}

[1] Probst C, Pape H-C, Hildebrand F, Regel G, Mahlke L, Giannoudis P, et al. 30 years of polytrauma care: an analysis of the change in strategies and results of 4849 cases treated at a single institution. Injury 2009;40:77-83.

[2] Allen GS, Coates NE. Pulmonary contusion: a collective review. Am Surg 1996:62:895-900.

[3] Wu J, Sheng L, Ma Y, Gu J, Zhang M, Gan J, et al. The analysis of risk factors of impacting mortality rate in severe multiple trauma patients with posttraumatic acute respiratory distress syndrome. Am J Emerg Med 2008;26:419-24.

[4] Wang S-H, Wei T-S, Chen C-P. Prognostic analysis of patients with blunt chest trauma admitted to an intensive care unit. J Formos Med Assoc Taiwan Yi Zhi 2007;106:444-51.

[5] Hoth JJ, Stitzel JD, Gayzik FS, Brownlee NA, Miller PR, Yoza BK, et al. The pathogenesis of pulmonary contusion: an open chest model in the rat. J Trauma 2006;61:32-44. discussion 44-45.

[6] Raghavendran K, Notter RH, Davidson BA, Helinski JD, Kunkel SL, Knight PR. Lung contusion: inflammatory mechanisms and interaction with other injuries. Shock Augusta Ga 2009;32:122-30.

[7] Moore FA, Sauaia A, Moore EE, Haenel JB, Burch JM, Lezotte DC. Postinjury multiple organ failure: a bimodal phenomenon. J Trauma 1996;40:501-10.

[8] Ahmad MA, Sante ED, Giannoudis PV. Assessment of severity of chest trauma: is there an ideal scoring system? Injury 2010;41:981-3.

[9] Gennarelli T, Wodzin E. The abbreviated injury scale. Assoc Adv Automot Med 2005;2005.

[10] Baker SP, O'Neill B, Haddon W, Long WB. The injury severity score: a method for describing patients with multiple injuries and evaluating emergency care. J Trauma 1974;14:187-96.
[11] Wagner RB, Jamieson PM. Pulmonary contusion. Evaluation and classification by computed tomography. Surg Clin North Am 1989;69:31-40.

[12] Pape H-C, Remmers D, Rice J, Ebisch M, Krettek C, Tscherne H. Appraisal of early evaluation of blunt chest trauma: development of a standardized scoring system for initial clinical decision making. J Trauma Acute Care Surg 2000; 49:496-504

[13] Mommsen P, Zeckey C, Andruszkow H, Weidemann J, Frömke C, Puljic P, et al. Comparison of different thoracic trauma scoring systems in regards to prediction of post-traumatic complications and outcome in blunt chest trauma. J Surg Res 2012;176:239-47.

[14] Hamada SR, Gauss T, Duchateau F-X, Truchot J, Harrois A, Raux M, et al. Evaluation of the performance of French physician-staffed emergency medical service in the triage of major trauma patients. J Trauma Acute Care Surg 2014;76:1476-83.

[15] Le Gall JR, Lemeshow S, Saulnier F. A new Simplified Acute Physiology Score (SAPS II) based on a European/North American multicenter study. J Am Med Assoc 1993;270:2957-63.

[16] Wagstaff TAJ, Soni N. Performance of six types of oxygen delivery devices at varying respiratory rates. Anaesthesia 2007;62:492-503.

[17] The ARDS Definition Task Force. Acute respiratory distress syndrome: the Berlin definition. J Am Med Assoc 2012;307:2526-33.

[18] Hernandez G, Fernandez R, Lopez-Reina P, Cuena R, Pedrosa A, Ortiz R, et al. Noninvasive ventilation reduces intubation in chest trauma-related hypoxemia: a randomized clinical trial. Chest J. 2010;137:74-80.

[19] Cannesson M, Le Manach Y, Hofer CK, Goarin JP, Lehot J-J, Vallet B, et al. Assessing the diagnostic accuracy of pulse pressure variations for the prediction of fluid responsiveness: a gray zone approach. Anesthesiology 2011;115: 231-41.

[20] Hoth J], Martin RS, Yoza BK, Wells JD, Meredith JW, McCall CE. Pulmonary contusion primes systemic innate immunity responses. J Trauma Inj Infect Crit Care 2009;67:14-22

[21] Murphy TJ, Paterson HM, Kriynovich S, Zang Y, Kurt-Jones EA, Mannick JA, et al. Linking the two-hit response following injury to enhanced TLR4 reactivity. J Leukoc Biol 2005;77:16-23.

[22] Simon B, Ebert J, Bokhari F, Capella J, Emhoff T, Hayward 3rd T, et al. Management of pulmonary contusion and flail chest: an Eastern Association for the Surgery of Trauma practice management guideline. J Trauma Acute Care Surg 2012;73:S351-61.

[23] Wutzler S, Wafaisade A, Maegele M, Laurer H, Geiger EV, Walcher F, et al. Lung Organ Failure Score (LOFS): probability of severe pulmonary organ failure after multiple injuries including chest trauma. Injury 2012;43:1507-12.

[24] Watkins TR, Nathens AB, Cooke CR, Psaty BM, Maier RV, Cuschieri J, et al. Acute respiratory distress syndrome after trauma: development and validation of a predictive model. Crit Care Med 2012;40:2295-303.

[25] McClure RJ, Peel N, Kassulke D, Neale R. Appropriate indicators for injury control? Public Health 2002;116:252-6.

[26] Miller PR, Croce MA, Bee TK, Qaisi WG, Smith CP, Collins GL, et al. ARDS after pulmonary contusion: accurate measurement of contusion volume identifies high-risk patients. J Trauma Acute Care Surg 2001;51:223-30.

[27] Youden WJ. Index for rating diagnostic tests. Cancer 1950;3:32-5.

[28] Ray P, Le Manach Y, Riou B, Houle TT. Statistical evaluation of a biomarker. Anesthesiology 2010;112:1023-40.

[29] Plurad D, Martin M, Green D, Salim A, Inaba K, Belzberg H, et al. The decreasing incidence of late posttraumatic acute respiratory distress syndrome: the potential role of lung protective ventilation and conservative transfusion practice. J Trauma 2007:63:1-7.

[30] Nahm NJ, Vallier HA. Timing of definitive treatment of femoral shaft fractures in patients with multiple injuries: a systematic review of randomized and nonrandomized trials. J Trauma Acute Care Surg 2012;73:1046-63.

[31] Chiumello D, Coppola S, Froio S, Gregoretti C, Consonni D. Noninvasive ventilation in chest trauma: systematic review and meta-analysis. Intensive Care Med 2013;39:1171-80.

[32] Gage A, Rivara F, Wang J, Jurkovich GJ, Arbabi S. The effect of epidural placement in patients after blunt thoracic trauma. J Trauma Acute Care Surg 2014:76:39-45.

[33] Pape H-C, Rixen D, Morley J, Husebye EE, Mueller M, Dumont C, et al. Impact of the method of initial stabilization for femoral shaft fractures in patients with multiple injuries at risk for complications (borderline patients). Ann Surg 2007;246:491-501 